\title{
A general slip-line field solution for the ultimate bearing capacity of a pipeline on drained soils
}

\author{
Fu-Ping Gao ${ }^{\mathrm{a}, *}$, Ning Wang ${ }^{\mathrm{a}}$, Bo Zhao ${ }^{\mathrm{a}, \mathrm{b}}$ \\ ${ }^{a}$ Key Laboratory for Mechanics in Fluid Solid Coupling Systems, Institute of Mechanics, Chinese Academy of Sciences, Beijing 100190, China \\ ${ }^{\mathrm{b}}$ CNPC Offshore Engineering Company Limited, Beijing 100028, China
}

\section{A R T I C L E I N F O}

\section{Article history:}

Received 12 November 2014

Accepted 20 May 2015

Available online 12 June 2015

Keywords:

Collapse loads

Bearing capacity

Slip-line field theory

Analytical solution

Submarine pipeline

\begin{abstract}
A B S T R A C T
An accurate evaluation of the ultimate bearing capacity of a cylindrical foundation is crucial for predicting pipe-soil interaction behaviors. A general slip-line field solution is derived for the ultimate bearing capacity of a pipeline on the drained soil obeying Mohr-Coulomb criterion. The slip-line field around the pipeline matches well with the corresponding plastic incremental-displacement field simulated by utilizing finite element analysis. Parametric studies indicate that as the internal friction angle of the soil approaches zero, the derived bearing capacity factors for the pipeline on the drained soil limit to those for the pipeline on the undrained soil obeying Tresca criterion. The bearing capacity factors for a fully-smooth pipeline then limit to those for a conventional rectangular strip-footing while the pipeline embedment approaches zero. Moreover, the dimensionless collapse load increases with increasing the pipeline embedment and the pipe-soil interfacial friction.
\end{abstract}

(c) 2015 Elsevier Ltd. All rights reserved.

\section{Introduction}

The ultimate bearing capacity of a foundation is the pressure causing shear failure of the supporting soil immediately below and adjacent to the foundation (see Knappett and Craig (2012)). Unlike conventional rectangular strip-footings, quite a few subsea structures, e.g., submarine pipelines, risers and mooring lines, hold circular sections. An efficient evaluation of the ultimate bearing capacity of a cylindrical foundation is crucial for predicting subsea structure-soil interaction behaviors, which may significantly affect the on-bottom stability of a submarine pipeline, the configuration of a steel catenary riser (SCR) at its touchdown zone, or the embedment of a circular mooring line into the seabed, etc. In the offshore engineering practices, the possibility for excessive settlement/sinking or floatation of such a subsea foundation should be checked in the design and maintenance stages (Det Norske, 2010).

The ultimate bearing capacity of a conventional shallow foundation with flat bottom on land has been investigated by applying slip-line (stress) field theory and upper-bound theorem of classical plasticity theory, revealing the failure mechanisms (Chen and Liu, 1990; Gourvenec and Randolph, 2003; Li et al., 2015). The bearing capacity of a strip-footing can be treated as a plane-strain problem. For a conventional strip-footing, Prandtl's solution has

\footnotetext{
* Corresponding author. Tel.: +8610 82544189; fax: +86 1062561284 .

E-mail address: fpgao@imech.ac.cn (F.-P. Gao).
}

been widely adopted to predict its bearing capacity. For the submarine pipeline holding a circular cross-section, some researchers, e.g. Small et al. (1971), predict its bearing capacity by the Prandtl's solution with some empirical corrections, i.e. using an equivalent width for certain value of the pipeline embedment. Nevertheless, this simplified treatment obviously could not well consider the effect of the circular section of pipeline foundations. Recently, the slip-line field solution for bearing capacity of a pipeline on clayey soils obeying Tresca failure criterion was derived by Gao et al. (2013). The parametric study indicated that the effect of circular section configuration on the bearing capacity factor gets more obvious with increasing dimensionless pipeline embedment $\left(e_{0} / r\right.$, where $e_{0}$ is the pipeline embedment into the seabed, and $r$ is the radius of the pipeline). That is, with the pipeline embedment $e_{0} / r$ increasing from zero (i. e. the pipeline just touching soil surface) to 1.0 (i.e., the pipeline being half buried), the bearing capacity factor for cohesion $\left(N_{c}\right)$ decreases from the value of " $2+\pi$ " to " 4.0 " accordingly. As such, if the pipeline foundation (with a circular-bottom) is simplified as a conventional strip footing (with a flat-bottom) without any corrections would over-evaluate the bearing capacity.

If undrained bearing capacity is being considered, the soil can be assumed to behave as a Tresca material; if drained bearing capacity is under investigation, the soil can then be regarded as a Mohr-Coulomb material (see Potts and Zdravković (2001)). Previous analytical solutions for the vertical bearing capacity of pipeline foundations are mainly for the purely cohesive soils 
obeying Tresca criterion, e.g., the upper and lower bound solutions by Murff et al. (1989); a slip-line field solution by Gao et al. (2013). Based on the plasticity theory and series of sideswipe tests of a partially embedded pipeline on calcareous sands, several pipe-soil interaction models (or named as force-resultant plasticity models) for the combined vertical and horizontal loading conditions have been successively developed and employed for simulating the pipeline on-bottom responses (e.g., Zhang et al., 2002; Tian and Cassidy, 2008; Hodder and Cassidy, 2010; Tian et al. 2010). As for the aforementioned pipe-soil interaction models, the behaviors of the entire pipe foundation were encapsulated by relating the resultant forces to the corresponding displacements. In the existing sideswipe or penetration tests, the loads on the pipe foundation were normally not beyond its vertical bearing capacity. The vertical bearing capacity is a critical value of the pressure inducing sudden settlement/collapse of the pipe foundation while penetrating into the soil, which is crucial for the vertical on-bottom stability of a submarine pipeline.

In this study, a general slip-line field solution is derived for the ultimate vertical bearing capacity of a pipeline on the drained soil obeying Mohr-Coulomb criterion, taking into account both the cohesion and the internal friction angle of the soil. The slip-line field for a pipeline on Mohr-Coulomb soils is constructed. Parametric studies are further conducted for understanding the failure mechanism of the pipeline foundation on drained soils.

\section{A general slip-line solution for the bearing capacity of $A$ pipeline foundation}

\subsection{Construction of the slip-line field for a pipeline on Mohr- Coulomb soils}

For a submarine pipeline laid on a topographically flat seabed, its length is typically much larger than the section dimension, thus the pipeline's bearing capacity is usually treated as a plane-stain problem. As aforementioned, the slip-line field for the cylindrical foundation or pipeline on Treaca soils has been constructed (Gao et al., 2012, 2013). In this section, a general slip-line field for the pipeline foundation on a Mohr-Coulomb soil will be further constructed.

As shown in Fig. 1, the pipeline with radius $r$ is laid on a seabed with an embedment $e_{0}$ and uniform surcharge pressure $q$ on the ground surface adjacent to the pipeline. Similar to the previous treatment for the surcharge pressures, in this study, the surcharge pressures $(q)$ are set as follows: (1) for the case of $e_{0} / r \leq 1, q$ is set to zero; (2) for the case of $e_{0} / r>1$, the pipeline embedment can be treated as $e_{0} / r=1$ with an equivalent uniform surcharge pressure $q=\gamma^{\prime}\left(e_{0}-r\right)$, where $\gamma^{\prime}$ is the submerged (buoyant) unit weight of the soil.

In the analytical study, the seabed is assumed as a homogenous, isotropic and perfectly-plastic material, obeying Mohr-Coulomb

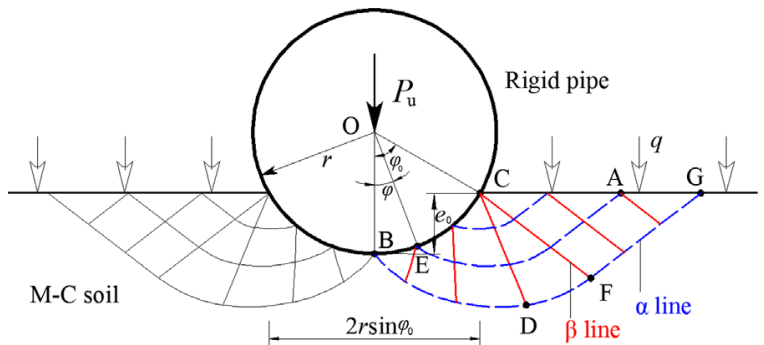

Fig. 1. The slip-line field of the pipeline foundation on a soil obeying MohrCoulomb failure criterion: a smooth pipe; internal frictional angle $\phi=15^{\circ}$. failure criterion. As well known, the Tresca criterion/model is for total stress analysis, and the Mohr-Coulomb criterion is for effective stress analyses. If the soil cohesion $(c)$ is replaced with the undrained shear strength (e.g., to simulate the undrained behavior of the saturated clayey soils) and the angle of shearing resistance $\phi$ is set to zero, the Mohr-Coulomb failure criterion can be degenerated to the Tresca criterion (see Potts and Zdravković (1999)).

The slip-line field is divided into three regions: the uniform region $\mathrm{CFG}$, the extrusion region $\mathrm{CBD}$ and the transition region $\mathrm{CDF}$ (see Fig. 1). The direction of $\alpha$ and $\beta$ slip-lines can be uniquely determined once the mean stress $\sigma$ (Note: $\sigma=\left(\sigma_{1}+\sigma_{3}\right) / 2$ ) and the shear stress (or normal stress) on an arbitrary direction are given. Under certain boundary conditions, the slip-line field could be constructed using characteristic functions of slip-line.

With the Riemann condition on CG and CEB, the stresses of the region $\mathrm{CFG}, \mathrm{CBD}$ and $\mathrm{CDF}$ can be calculated successively. The soil within the slip-line field is supposed to be in critical failure condition. The direction of the third principle stress is vertical in the uniform region CFG. Given the value of the uniform load $q$, the stresses in field CFG and along the boundary CF can be solved. The field CBD is the extrusion region. The direction of the first principle stress at line CEB is radial for a purely smooth pipe. Point $\mathrm{E}$ at the boundary CEB are connected with $\mathrm{CF}$ by $\alpha$ slip-line and the shear stress can be determined if interfacial friction $f$ is properly defined, the details of which is given in Section 2.2. Therefore, the stresses on the boundary CEB is determined using the characteristic functions. Then the stresses in the field CDB and line CD can be obtained, identically using the characteristic functions. Thus, CDF can be determined according to the stresses at $\mathrm{CF}$ and $\mathrm{CD}$. By adopting a finite difference approximation, the whole slip-line field can be constructed (as shown in Fig. 1). Noting that, in the construction of the slip-line field for a Mohr-Coulomb soil, the soil cohesion can be arbitrary, i.e. the magnitude of the slip-line field is not related to the value of soil cohesion. The $\beta$ and $\alpha$ lines in the uniform region (CFG) are straight lines while the $\alpha$ lines are curved in the extrusion region $\mathrm{CBD}$ and the transition region CDF.

\subsection{Collapse load: slip-line field solution for Mohr-Coulomb soils}

The collapse load $\left(P_{u}\right)$ for the ultimate bearing capacity of a pipeline foundation can be expressed with the integral of the stresses along the pipe-soil interface as follows:

$P_{u}=2 \int_{0}^{\varphi_{0}} r \sigma_{E, y} d \varphi$.

In which $\sigma_{E, y}$ is the vertical component of the pipe-soil interfacial force; $\varphi_{0}$ is the embedment angle $\angle \mathrm{BOC}$ (see Fig. 1), i.e. $\varphi_{0}=\arccos \left(1-e_{0} / r\right) ; \varphi=\angle \mathrm{BOE}$ is an arbitrary angle for the integration along the pipe-soil contact boundary. Based on the slip-line field theory, the stress equation along the $\alpha$ line for the soil obeying MohrCoulomb criterion is derived as (see Appendix A):

$\sigma_{0} e^{-2 \omega \tan \phi}=$ const 1

As shown in Fig. 1, the points A and E are along the same $\alpha$ line. Thus the following relationships exist for the stresses at point $\mathrm{A}$ and $\mathrm{E}$, i.e.

$\sigma_{0 E} e^{-2 \omega_{E} \tan \phi}=\sigma_{0 A} e^{-2 \omega_{A} \tan \phi}$,

In which, $\sigma_{0}=c \cot \phi+\sigma$, where $c$ and $\phi$ are the cohesion and internal friction angle of the soil respectively; $\sigma\left(=\left(\sigma_{1}+\sigma_{3}\right) / 2\right)$ is the mean stress in the soil; $\omega$ is the angle from the $x$-axis to the first principal stress plane in the clockwise direction (see Fig. 9).

The point-A is along the soil surface, $\omega_{A}=\pi / 2$ and $\sigma_{0 A}=(q+c \cot \phi) /(1-\sin \phi)$. Fig. 2 shows the Mohr-circle for 


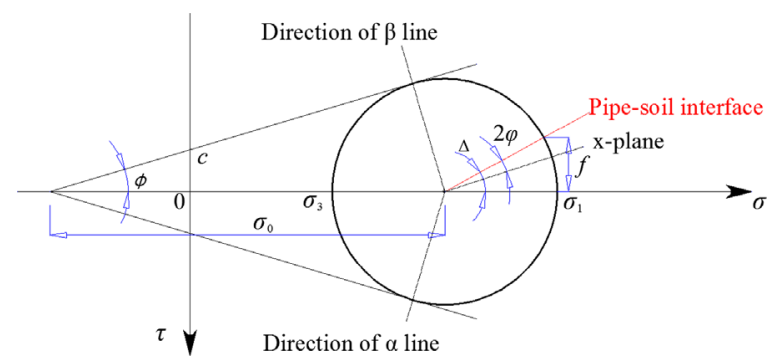

Fig. 2. Mohr-circle for point-E along the pipe-soil interface.

the point-E. The angle from the $\mathrm{x}$-axis to the first principal stress plane at the point-E

$\omega_{E}=\pi-\varphi+\Delta / 2$,

In which, $\Delta / 2$ is the angle from the tangent of the pipe-soil interface to the first principal stress plane (clockwise), $\Delta=$ $\arcsin \left[f /\left(\sigma_{0 E} \sin \varphi\right)\right]$. Note: the subscript " $E$ " or " $A$ " denotes the value of the labeled parameter at the point-E or point-A, respectively. Submitting the values of $\omega_{A}, \sigma_{O A}$ and $\omega_{E}$ into Eq. (2'), $\sigma_{0 E}$ can be obtained as:

$\sigma_{0 E}=\frac{q+c \cot \phi}{1-\sin \phi} \exp [(\pi-2 \varphi+\Delta) \tan \phi]$.

The vertical component of the pipe-soil contact force $\left(\sigma_{E, y}\right)$ can be expressed as:

$\sigma_{E, y}=\tau \sin \varphi+\sigma \cos \varphi$,

i.e.

$\sigma_{E, y}=\sigma_{0 \mathrm{E}} \sin \varphi \sin \Delta \sin \phi+\cos \phi\left(\sigma_{0 \mathrm{E}}-c \cot \varphi+\sigma_{0 \mathrm{E}} \sin \varphi \cos \Delta\right)$.

The definition of the pipe-soil friction $(f)$ is needed to determine the value of $\Delta$, half value of which is the clockwise angle from the tangent of the pipe-soil interface to the first principal stress plane. For the pipe laid on a Mohr-Coulomb soil, the pipesoil friction $f$ is defined as:

$f=\mu_{a} \sigma_{0}$,

where $\mu_{a}$ is an apparent friction coefficient for the pipe-soil interface, which is physically relative with the roughness of the pipe's surface, the particle diameter of the neighboring soils, etc.; $\sigma_{0}=c \cot \phi+0.5\left(\sigma_{1}+\sigma_{3}\right)$, indicating the soil cohesion, internal friction angle and the mean effective principal stress are taken into account. As $\Delta=\arcsin \left[f /\left(\sigma_{0} \sin \varphi\right)\right]$ (see Fig. 2), the value of $\Delta$ can then be calculated with

$\Delta=\arcsin \left(\frac{\mu_{a}}{\sin \phi}\right)$.

Referring to Fig. 2, the values of pipe-soil friction $f$ are not larger than the radius of the Mohr circle $\left(\sigma_{0} \sin \phi\right)$, i.e.

$\frac{\mu_{a}}{\sin \phi} \leq 1.0$.

Submitting Eqs. (6) and (8) into Eq. (1), the collapse load for the pipeline foundation can be derived as:

$$
\begin{aligned}
P_{\mathrm{u}}= & \frac{2 r(q+c \cot \phi)}{(1-\sin \phi)\left(1+4 \tan ^{2} \phi\right)}\left\{\left[-\sin \phi \sin \Delta\left(2 \tan \phi \sin \varphi_{0}+\cos \varphi_{0}\right)\right.\right. \\
& \left.+(1+\sin \phi \cos \Delta)\left(\sin \varphi_{0}-2 \tan \phi \cos \varphi_{0}\right)\right] e^{\left(\pi-2 \varphi_{0}+\Delta\right) \tan \phi} \\
& +[\sin \phi \sin \Delta+2(1+\sin \phi \cos \Delta) \tan \phi] e^{(\pi+\Delta) \tan \phi} \\
& -2 r c \cot \phi \sin \varphi_{0}
\end{aligned}
$$

Referring to the bearing capacity of strip footings (see Knappett and Craig (2012)), the bearing capacity of the pipeline foundation on a Mohr-Coulomb soil can be expressed as:

$\frac{P_{u}}{2 r \sin \varphi_{0}}=c N_{c}+q N_{q}+\gamma^{\prime} r \sin \varphi_{0} N_{\gamma}$,

where " $2 r \sin \varphi_{0}$ " is the width of the pipe-soil interface (see Fig. 1). The bearing capacity factors are derived theoretically assuming the soil is weightless (i.e. $\gamma^{\prime}=0$ ). Submitting Eq. (9) into Eq. (10), the bearing capacity factor for the cohesion $\left(N_{c}\right)$ and that for the distributed load $\left(N_{q}\right)$ can thereby be obtained as follows:

$$
\begin{aligned}
N_{c}= & \frac{\cot \phi}{\sin \varphi_{0}(1-\sin \phi)\left(1+4 \tan ^{2} \phi\right)}\left\{\left[-\sin \phi \sin \Delta\left(2 \tan \phi \sin \varphi_{0}+\cos \varphi_{0}\right)\right.\right. \\
& \left.+(1+\sin \phi \cos \Delta)\left(\sin \varphi_{0}-2 \tan \phi \cos \varphi_{0}\right)\right] e^{\left(\pi-2 \varphi_{0}+\Delta\right) \tan \phi} \\
& +[\sin \phi \sin \Delta+2 \tan \phi(1+\sin \phi \cos \Delta)] e^{(\pi+\Delta) \tan \phi} \\
& \left.-\sin \varphi_{0}(1-\sin \phi)\left(1+4 \tan ^{2} \phi\right)\right\} \\
N_{q}= & N_{c} \tan \phi+1 .
\end{aligned}
$$

Due to the complexity of the governing hyperbolic partial differential equations of the slip-line field theory, it is rarely possible to obtain analytical stress field solutions for a frictional soil (i.e. $\phi>0$ ) which has weight. As well known, $N_{\gamma}$ has been obtained from approximate calculations assuming the soil has weight, but no cohesion and surcharge. One of the most popular expressions for $N_{\gamma}$ proposed by Hansen (1970) is adopted (see Knappett and Craig (2012)):

$N_{\gamma}=1.80\left(N_{q}-1\right) \tan \phi$

As with many of the alternatives, this expression can be used for both rough and smooth footings (Potts and Zdravković, 2001).

\section{Comparison with numerical simulation}

The bearing capacity of the pipeline foundation on the horizontally flat soil can be regarded as a symmetric plane-strain problem. A symmetrical plane-strain finite element model is used to simulate the vertically-loaded pipe-soil interaction (see Fig. 3). As the stiffness of a steel pipeline is much larger than that of the soil, the pipe is assumed to be rigid in this numerical simulation. The plasticity characteristics of the seabed sediments may result in

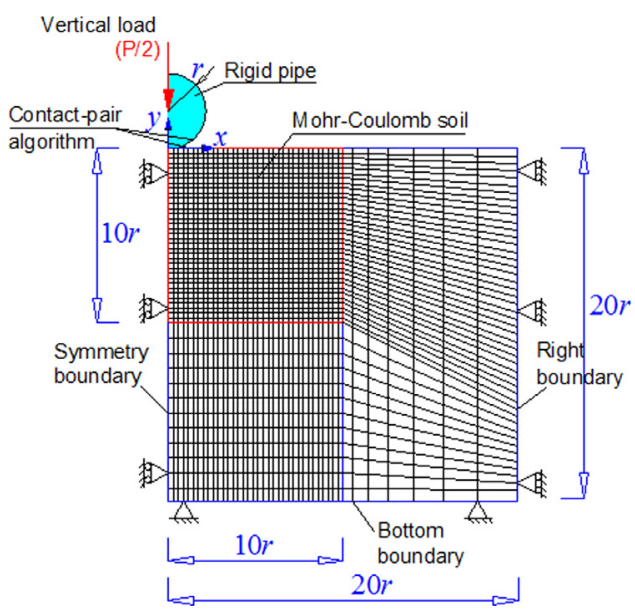

Fig. 3. A symmetrical plane-strain FE model for the vertically-loaded pipe-soil interaction: illustration of the meshes and boundary conditions. 
significant effects on the bearing capacity of the pipeline foundation. Similar with the basic assumptions in the slip-line field analysis, the elasto-plastic Mohr-Coulomb model is used in the finite element simulation of the elasto-plastic behavior of the sediments, especially when the drained bearing capacity of a foundation is under investigation. The yield function of MohrCoulomb model is written as (see Knappett and Craig (2012)):

$J-\left(\frac{c}{\tan \phi}+p^{\prime}\right) g(\theta)=0$

in which, $J$ is the deviatoric stress invariant; $p^{\prime}$ is the mean effective stress

$g(\theta)=\frac{\sin \phi}{\cos \theta+\sin \theta \sin \phi / \sqrt{3}}$,

where $\theta$ is the Lode's angle.

The pipe-soil interface changes while the pipeline penetrating into the soil, thus the interfacial behavior is vitally important to efficiently simulate the vertical pipe-soil interaction. As shown in Fig. 3, the contact-pair algorithm provided in the ABAQUS software (Hibbitt and Sorensen, 2006) is employed for simulating the contacting boundary between the partially-embedded pipeline and the neighboring soil; and the left non-contact surface of the seabed is regarded as a free boundary. The vertical pipe-soil interaction is a symmetric plane-strain problem. As such, the left side is regarded as an axisymmetric boundary, i.e. both the rotational and the translational degree of freedom in the horizontal $(x)$ direction are restrained. The right side of the mesh is also restrained in the horizontal direction. At the bottom of the model, the translational degrees of freedom in both $x$ and $y$ directions are fixed. The FE mesh gets more refined at closer proximity to the pipeline, so as to obtain high calculation efficiency. Following grid analyses, the width of numerical model is chosen as $20 \mathrm{r}$ and the depth as 20r. In the FE model, the elasticity modulus of the soil $E=1.0 \mathrm{MPa}$; mass density $\rho=1.8 \times 10^{3} \mathrm{~kg} / \mathrm{m}^{3}$; Poisson ratio $\nu=0.3$ for a drained sandy soil, $\nu=0.48$ for an undrained clayey soil; the radius of the rigid pipe $r=0.25 \mathrm{~m}$. The values of the soil cohesion $c$ and the internal friction angle $\phi$ are various for the comparison with the slip-line field solution.

The failure mechanism for a shallow foundation can be interpreted by the incremental-displacement vector field in the numerical simulation (see Potts and Zdravković, 2001; McMahon et al., 2014). Fig. 4(a) and (b) shows the incremental-displacement vector field numerically simulated with the above finite element model and the slip-line field constructed with the newly derived theoretical solution, respectively, for a smooth pipeline laid on an undrained clayey soil $(\phi \rightarrow 0)$ with $c \approx 5 \mathrm{kPa}$, which is suffering a general shear failure. The orientations of these vectors (see Fig. 4 (a)) indicating the directions of soil movement match well with the $\alpha$ lines of the slip-line field solution (see Fig. 4(b)).

Similarly, Fig. 5(a) and (b) shows the plastic incrementaldisplacement vector field simulated with the numerical model and the slip-line field constructed with the theoretical solution, respectively, for a smooth pipeline laid on a Mohr-Coulomb soil with $c \approx 5 \mathrm{kPa}, \phi=18^{\circ}$. The orientations of the plastic incremental-displacement vectors (see Fig. 5(a)) match well with the $\alpha$ lines of the slip-line field solution (see Fig. 5(b)). Comparison between Fig. 4 (for $\phi \rightarrow 0$ ) and Fig. 5 (for $\phi=18^{\circ}$ ) indicates that, an increase of the internal friction angle of the soil enlarges the magnitude of the slip-line field and eventually increases the bearing capacity of the pipeline foundation. The effects of internal friction angle will be further investigated in Section 4 .

To make a quantitative comparison between the newly derived slip-line solutions and the numerical results, the dimensionless collapse load $P_{u} / \mathrm{cr}$ is employed. Then Eq. (10) for the bearing capacity of a pipeline on Mohr-Coulomb soils can be expressed
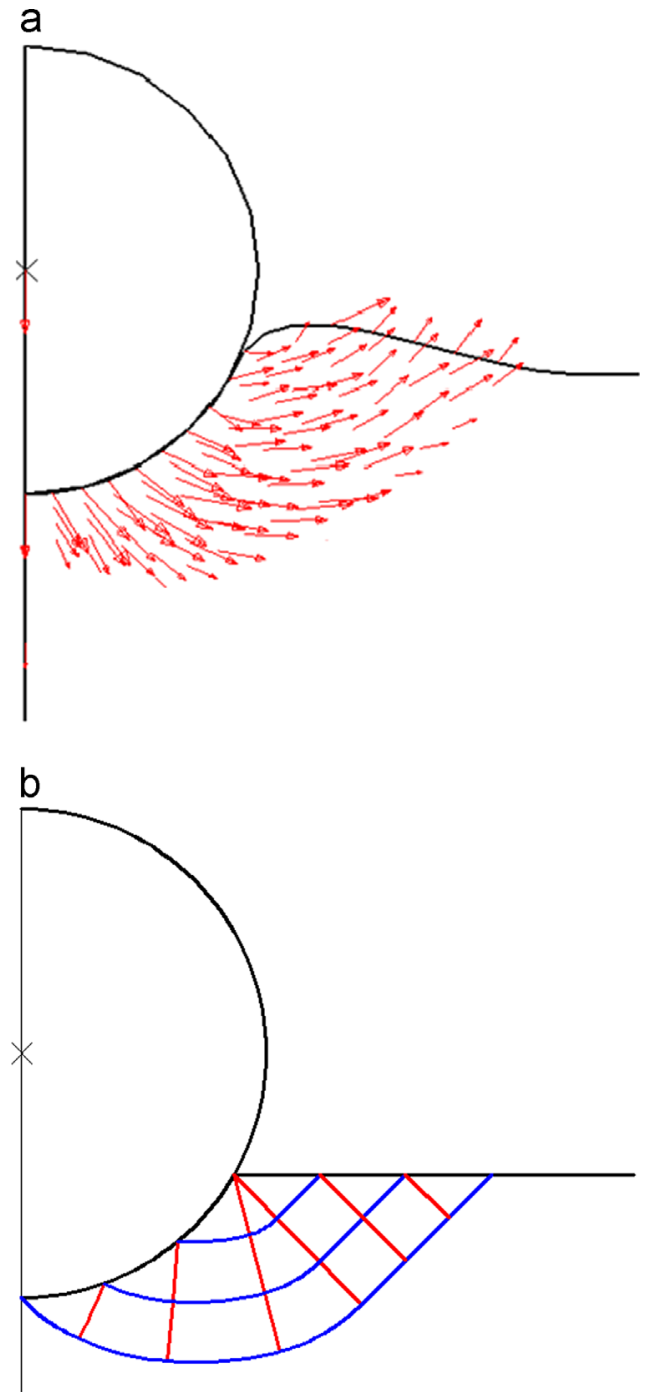

Fig. 4. (a) Plastic incremental-displacement vector field simulated with the numerical model; (b) Slip-line field constructed with the theoretical solution for a smooth pipeline: $c \approx 5 \mathrm{kPa}, \phi=0$

with non-dimensional form as follows:

$P_{u} / c r=2\left(N_{c}+\frac{q}{c} N_{q}+\frac{\gamma^{\prime} r \sin \varphi_{0}}{c} N_{\gamma}\right) \sin \varphi_{0}$

Table 1 shows the results of the collapse load with finite element modeling (denoted as $P_{\mathrm{ufE}}$ ) and those of the slip-line field solution (denoted as $P_{\mathrm{uSL}}$ ) for various values of soil parameters, respectively. In this comparison, the conditions of weightless soil $\left(\gamma^{\prime}=0\right)$ and no surcharge $(q=0)$ are taken into account for the simplification purpose. Fig. 6 shows the variation of the dimensionless ultimate loads $\left(P_{u} / c r\right)$ with the dimensionless pipeline embedment/settlement $\left(e_{0} / r\right)$ for various values of $\phi$. The comparison between the slip-line field solutions and those of finite element modeling indicates that they match well with similar trends for the variation of $P_{u} / c r$ with $e_{0} / r$. For a given value of $\phi$ (e.g., $\phi=5^{\circ}, 10^{\circ}, 15^{\circ}$ ), $P_{u} /$ crincreases gradually with the increase of $e_{0} / r$. Note that, the pipeline settlement in the elastoplastic FE modeling involves both the elastic-deformation component and the plastic-deformation one of the neighboring soils; but in the slip-line field solutions, only plastic-deformation are involved. As such, the numerical results are slightly higher than those of the slip-line field solutions for the same values of $e_{0} / r$ and $\phi$ (see Fig. 6). The relative difference $\left(P_{\mathrm{uSL}}-P_{\mathrm{uFE}}\right) / P_{\mathrm{uSL}}$ is within $15 \%$ for the examined soil parameters (also see Table 1 ). 


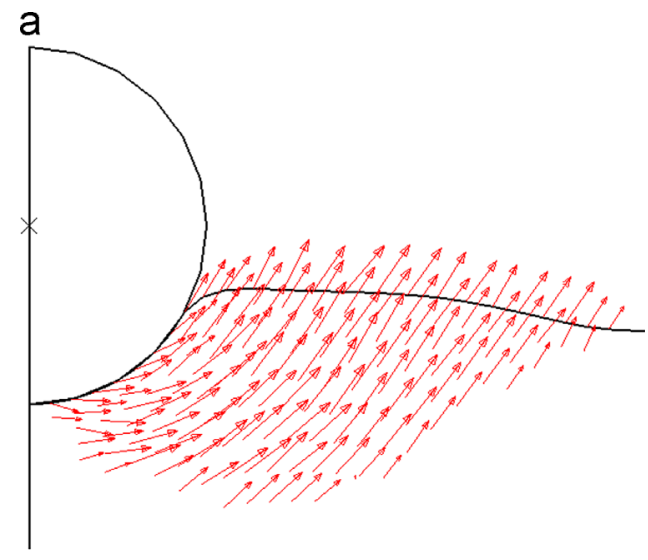

b

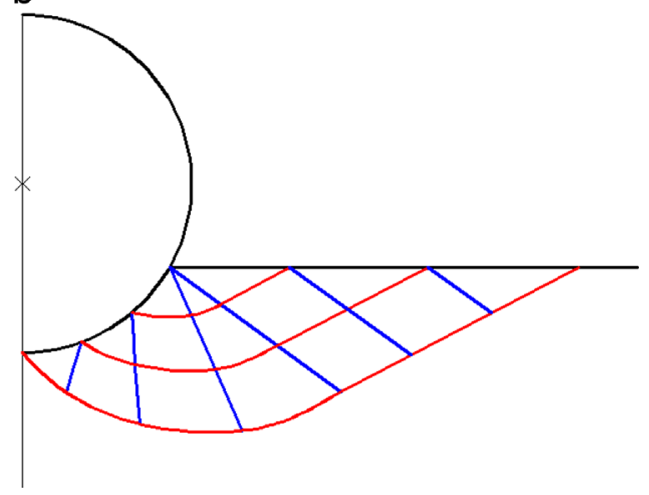

Fig. 5. (a) Plastic incremental-displacement vector field simulated with the numerical model; (b) Slip-line field constructed with the theoretical solution for a smooth pipeline: $c \approx 5 \mathrm{kPa}, \phi=18^{\circ}$.

Table 1

Numerical results and slip-line field solutions for the collapse loads for a smooth pipeline on Mohr-Coulomb soils.

\begin{tabular}{lccccccc}
\hline$e_{0}(\mathrm{~m})$ & $e_{0} / r$ & $c(\mathrm{kPa})$ & $\phi(\circ)$ & $P_{u \mathrm{FE}}(\mathrm{kN} / \mathrm{m})$ & $P_{u \mathrm{FE}} / c r$ & $P_{u \mathrm{SL}} / c r$ & $\frac{P_{u \mathrm{SL}}-P_{u \mathrm{FE}}}{P_{u \mathrm{SL}}}$ \\
\hline 0.083 & 0.33 & 5 & 5 & 9.5 & 7.60 & 7.91 & $3.9 \%$ \\
0.212 & 0.85 & 10 & 5 & 23.1 & 9.24 & 9.58 & $3.5 \%$ \\
0.198 & 0.79 & 15 & 5 & 33.5 & 8.93 & 9.53 & $6.3 \%$ \\
$0.210^{\mathrm{a}}$ & $0.84^{\mathrm{a}}$ & $20^{\mathrm{a}}$ & $5^{\mathrm{a}}$ & $43.2^{\mathrm{a}}$ & $8.64^{\mathrm{a}}$ & - & - \\
0.095 & 0.38 & 5 & 10 & 11.5 & 9.20 & 10.16 & $9.4 \%$ \\
0.146 & 0.58 & 10 & 10 & 24.8 & 9.61 & 11.19 & $14.1 \%$ \\
0.226 & 0.90 & 15 & 10 & 39.8 & 9.92 & 11.75 & $15.6 \%$ \\
$0.226^{\mathrm{a}}$ & $0.90^{\mathrm{a}}$ & $20^{\mathrm{a}}$ & $10^{\mathrm{a}}$ & $50.0^{\mathrm{a}}$ & $10.00^{\mathrm{a}}$ & - & - \\
0.107 & 0.43 & 5 & 15 & 14.1 & 11.52 & 13.14 & $12.3 \%$ \\
0.190 & 0.76 & 10 & 15 & 31.0 & 12.40 & 14.44 & $14.1 \%$ \\
0.240 & 0.96 & 15 & 15 & 47.4 & 12.64 & 14.63 & $13.6 \%$ \\
\hline
\end{tabular}

a Represents the numerical results for the pipeline foundation suffering punching shear failure.

A parametric study on the bearing capacity factors is for the pipeline foundation on Mohr-Coulomb soils will be further made in Section 4.

\section{Parametric study on bearing capacity factors}

4.1. Bearing capacity factors: comparison between Mohr-Coulomb solution and Tresca solution

In Section 2.2, the derived general solutions of the bearing capacity factors is for the pipeline foundation on Mohr-Coulomb

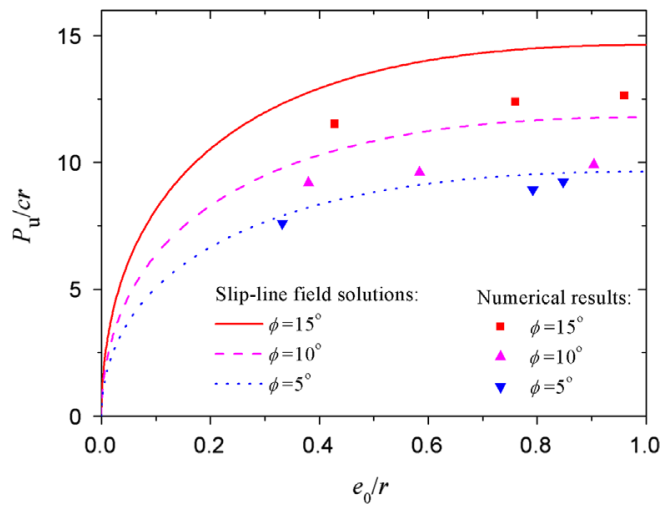

Fig. 6. Comparison between slip-line field solutions and numerical results: variation of $P_{u} / c r$ with $e_{0} / r$ for various values of $\phi$.

soils, i.e. both the soil cohesion and the internal friction angle are taken into account. While the internal friction angle approaches zero, the newly derived slip-line field solution for a pipeline foundation on Mohr-Coulomb soils should limit to that for Tresca soils. The derivation details are given as follows:

The bearing capacity factor for the cohesion $N_{c}$ (Eq. (11a)) can be expressed as:

$N_{c}=\frac{A_{1}+A_{2}+A_{3}}{\sin \varphi_{0}(1-\sin \phi)\left(1+4 \tan ^{2} \phi\right)}$

in which,

$A_{1}=4 \tan \phi \sin \phi \sin \varphi_{0}-4 \tan \phi \sin \varphi_{0}+2 \sin \phi \cos \Delta e^{(\pi+\Delta) \tan \phi}$

$-2 \sin \phi\left(\sin \Delta \sin \varphi_{0}+\cos \Delta \cos \varphi_{0}\right) e^{\left(\pi-2 \varphi_{0}+\Delta\right) \tan \phi}$,

$A_{2}=\cot \phi \sin \varphi_{0}\left(e^{\left(\pi-2 \varphi_{0}+\Delta\right) \tan \phi}-1\right)$,

$A_{3}=\left(\cos \phi \cos \Delta \sin \varphi_{0}-\cos \phi \sin \Delta \cos \varphi_{0}-2 \cos \varphi_{0}\right) e^{\left(\pi-2 \varphi_{0}+\Delta\right) \tan \phi}$

$+(\cos \phi \sin \Delta+2) e^{(\pi+\Delta) \tan \phi}+\cos \phi \sin \varphi_{0}$.

As the internal friction angle approaches zero $(\phi \rightarrow 0)$, the extrema for $A_{1}, A_{2}$ and $A_{3}$ are obtained as:

$\lim _{\phi \rightarrow 0} A_{1}=0$

$\lim _{\phi \rightarrow 0} A_{2}=\lim _{\phi \rightarrow 0} \frac{\sin \varphi_{0} e^{\left(\pi-2 \varphi_{0}+\Delta\right) \tan \phi} \sec ^{2} \phi\left(\pi-2 \varphi_{0}+\Delta\right)}{\sec ^{2} \phi}=\sin \varphi_{0}\left(\pi-2 \varphi_{0}+\Delta\right)$,

$\lim _{\phi \rightarrow 0} A_{3}=\sin \varphi_{0}-\sin \Delta \cos \varphi_{0}+\cos \Delta \sin \varphi_{0}-2 \cos \varphi_{0}+\sin \Delta+2$.

It is worth noting that the L'Hôpital's rule is used in the calculation the limit in (16b). Submitting Eqs. (16a)-(16c) into the limit expression of (14), and meanwhile calculating the limit of (11-b), then

$\lim _{\phi \rightarrow 0} N_{c}=\frac{\sin \Delta\left(1-\cos \varphi_{0}\right)-2\left(\cos \varphi_{0}-1\right)}{\sin \varphi_{0}}-2 \varphi_{0}+\cos \Delta+\Delta+\pi+1$,

$\lim _{\phi \rightarrow 0} N_{q}=1$,

which matches exactly the slip-line field solution for a pipeline foundation on Tresca soils previously obtained by the Gao et al. 
(2013), i.e.

$$
\begin{aligned}
N_{c}= & \frac{\sin \Delta\left(1-\cos \varphi_{0}\right)-2\left(\cos \varphi_{0}-1\right)}{\sin \varphi_{0}} \\
& +1+\Delta+\pi+\cos \Delta-2 \varphi_{0} \text { (Trasca solution), }
\end{aligned}
$$

$N_{q}=1$ (Trasca solution)

For the case of the partially-embedded pipeline laid on Tresca soils, if the pipeline surface is fully-smooth $(\Delta=0)$, then the two extrema of the bearing capacity factor $N_{c}$ (see (16a)): $\lim N_{c}=$ $2+\pi ; \lim _{\varphi_{0} \rightarrow \frac{\pi}{2}} N_{c}=4$.

Fig. 7 shows the variation of the bearing capacity factors $N_{c}$ and $N_{q}$ with the internal frictional angle $\phi$ for various values of the embedment ratio $e_{0} / r$ of a smooth pipeline, i.e. $e_{0} / r=0,0.13,0.50,1.00$ (the corresponding embedment angle $\varphi_{0}=0,30^{\circ}, 45^{\circ}, 60^{\circ}, 90^{\circ}$, respectively). It is indicated that the internal friction angle of the soil has much effect on the bearing capacity factors. The value of $N_{c}$ increases with the increase of the soil's internal friction angle. Under the condition of the internal friction angle of the soil equals zero $(\phi=0)$, the value of $N_{c}$ and $N_{q}$ for the pipe embedment approaching zero and that for half burial are as follows: $N_{c}=2+\pi$ (when $e_{0} / r=0$ ), $N_{c}=4$ (when $e_{0} / r=1.0$ ); $N_{q}=1$ (when $e_{0} / r$ is arbitrary), which matches well the slip-line field solution for the smooth pipeline on Tresca soils (see Eqs. (18a) and (18b)). For the present slip-line field solution for Mohr-Coulomb soils, let the apparent friction coefficient for the pipesoil interface $\mu_{a}=\alpha \sin \phi$, then $\Delta=\arcsin \alpha$ (see Eq. (8)), being the same definition as that in the slip-line field solution for a pipeline foundation on Tresca soils. As for a pipeline is laid on the purelycohesive soils or a clayey soil under undrained condition (the internal

\section{a}

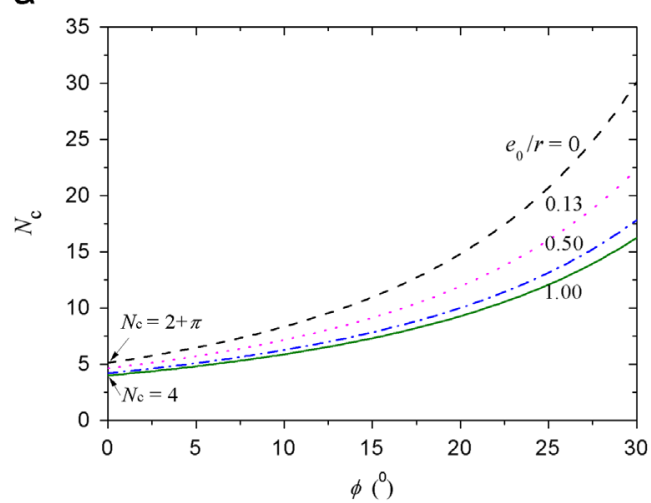

b

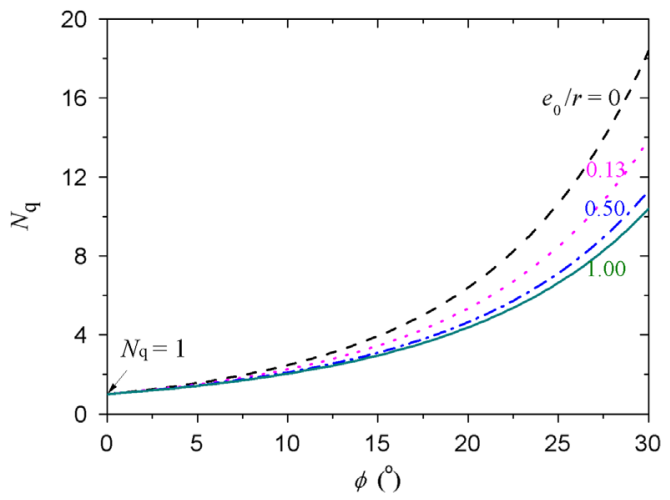

Fig. 7. Variation of bearing capacity factors with internal frictional angle for various values of the embedment of a smooth pipeline: (a) $N_{c}-\phi$, (b) $N_{q}-\phi$. friction angle $\phi \rightarrow 0$ ), the pipe-soil interfacial friction (tangent) would vanish (see Eq. (7)).

\subsection{Bearing capacity factors: comparison with those for a conventional strip footing}

To further compare with the existing solution for a conventional strip footing with flat bottom would be helpful for understanding the bearing capacity of the pipeline foundation. For the general shearing failure mechanism of a conventional strip footing, the Prandtl-Reissner solution has been widely employed. The ultimate bearing capacity of a rectangular strip footing on the surface of a weightless soil is expressed as:

$\frac{P_{u}}{b}=c N_{c}+q N_{q}$ (for a conventional strip footing),

in which, $P_{u}$ and $b$ are the ultimate load and the width of the footing, respectively; $N_{c}$ and $N_{q}$ are the corresponding bearing capacity factors for the conventional strip footing:

$N_{c}=\left(N_{q}-1\right) \cot \phi$,

$N_{q}=e^{\pi \tan \phi} \tan ^{2}\left(\frac{\pi}{4}+\frac{\phi}{2}\right)$

In this section, a degeneration analysis is made on the derived slip-line field solution for Mohr-Coulomb soils. Submitting (11a) into (11b), the bearing capacity factor of distributed load $\left(N_{q}\right)$ can be rewritten as:

$$
\begin{aligned}
N_{q}= & \frac{1}{(1-\sin \phi)\left(1+4 \tan ^{2} \phi\right)}\{(-2 \tan \phi \sin \phi \sin \Delta \\
& +\sin \phi \cos \Delta+1) e^{\left(\pi-2 \varphi_{0}+\Delta\right) \tan \phi} . \\
& +[\sin \phi \sin \Delta+2 \tan \phi(\sin \phi \cos \Delta+1)] \\
& {\left.\left[e^{(\pi+\Delta) \tan \phi}-\cos \varphi_{0} e^{\left(\pi-2 \varphi_{0}+\Delta\right) \tan \phi}\right] / \sin \varphi_{0}\right\} }
\end{aligned}
$$

As the pipeline embedment angle approaches zero $\left(\varphi_{0} \rightarrow 0\right)$, the limit of $N_{q}$ is then derived as:

$$
\begin{aligned}
\lim _{\varphi_{0} \rightarrow 0} N_{q} & =\frac{e^{(\pi+\Delta) \tan \phi}}{(1-\sin \phi)\left(1+4 \tan ^{2} \phi\right)}(\sin \phi \cos \Delta+1)\left(1+4 \tan ^{2} \varphi\right) \\
& =e^{(\pi+\Delta) \tan \varphi} \frac{\sin \phi \cos \Delta+1}{1-\sin \phi} .
\end{aligned}
$$

For a fully-smooth pipeline $(\Delta=0)$, Eq. (22) can be further simplified as:

$\lim _{\varphi_{0} \rightarrow 0} N_{q}=e^{\pi} \tan \phi \tan ^{2}\left(\frac{\pi}{4}+\frac{\phi}{2}\right)$ (for a smooth pipeline),

Which matches exactly the bearing factor of the distributed load for the conventional strip footing (i.e. (20b)). Similarly, the expressions of the bearing factor of the soil cohesion $\left(N_{c}\right)$ are same for both fully-smooth pipeline and the conventional strip footing while $\varphi_{0} \rightarrow 0$, as the same correlation exists between $N_{c}$ and $N_{q}$ (see (11b) or (20a)).

Fig. 8 shows the variation of bearing capacity factors with the foundation embedment for various values of the internal friction angle of the soil, i.e. $\phi=0,15^{\circ}$ and $30^{\circ}$. While the dimensionless pipeline embedment $\left(e_{0} / r\right)$ decreasing from the value of 1.0 (the pipeline being half-buried) to zero (the pipeline just touching the soil surface), the values of both $N_{c}$ and $N_{q}$ increase gradually to the solutions for a conventional strip-footings for the same value of the internal friction angle of the soil $(\phi)$. That is, the bearing capacity factors $N_{c}$ and $N_{q}$ for the smooth pipe as its embedment approaching zero are equal to the well-known Prandtl-Reissner solution for the conventional smooth strip footing. For a given value of $e_{0} / r$, the increase of $\phi$ brings an obvious increase of the 
two bearing capacity factors $\left(N_{c}\right.$ and $\left.N_{q}\right)$, especially for the shallowly-embedded pipelines. As shown in Fig. 8, when choosing $\phi=30^{\circ}$, if the half-buried pipeline foundation $\left(e_{0} / r=1.0\right)$ is treated as a conventional rectangular strip footing, the bearing capacity factors of the pipeline would be over evaluated, whose values are only $54 \%$ for $N_{c}$, and $57 \%$ for $N_{q}$ of the strip-footing, respectively.

\subsection{Effects of pipe-soil interfacial friction}

As discussed in Section 2.1, the directions of slip-lines in the extrusion zone $\mathrm{CBD}$ are relative with the pipe-soil interfacial friction. In this section, the effects of pipe-soil interfacial friction are examined.

a

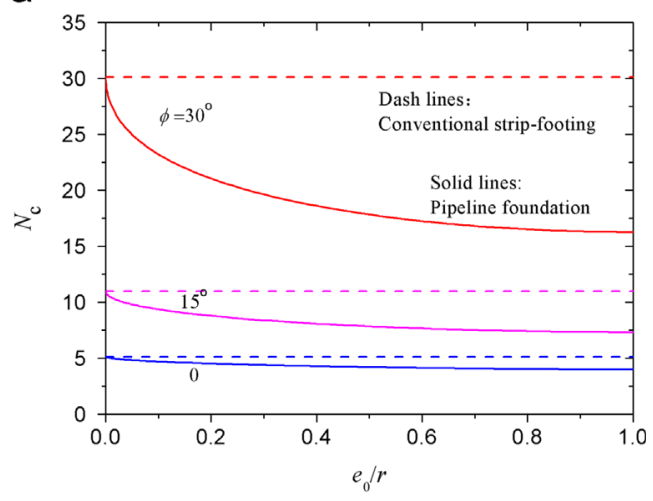

b

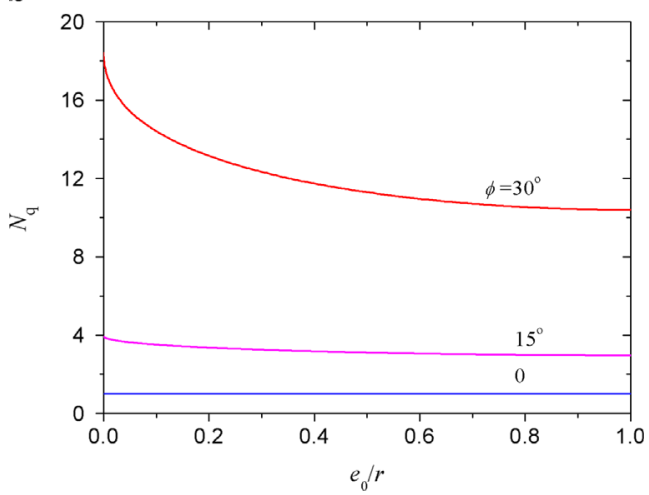

Fig. 8. Variation of bearing capacity factors with foundation embedment for various values of the internal frictional angle of the soil (solid lines: a smooth pipeline; dash lines: a conventional smooth strip-footing): (a) $N_{c}-e_{0} / r$, (b) $N_{q}-e_{0} / r$.

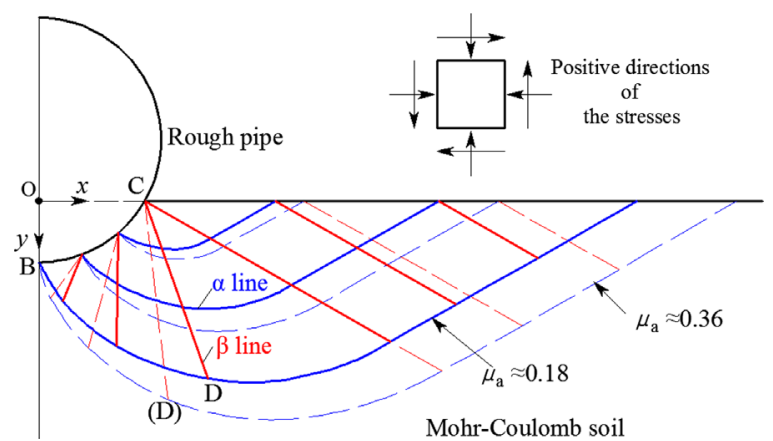

Fig. 9. Construction of slip-line fields of rough pipelines with various values of $\mu_{a}$ on a Mohr-Coulomb soil $\left(\phi=30^{\circ}\right)$.
In the expressions for the bearing capacity factors, i.e. (11a)(11c), besides the soil cohesion, the internal friction angle of the soil, and the pipeline embedment etc., the pipe-soil interfacial friction is also involved. According to the slip-line field theory (see Section 2.1 and Appendix A), the slip-line fields of the pipeline foundation are constructed for the rough pipes with various values of pipe-soil interfacial friction $\mu_{a}$ (e.g., $\mu_{a}=0.18,0.36$ ) on a MohrCoulomb soil with $\phi=30^{\circ}$ (see Fig. 9). It is shown that, with increasing the pipe-soil contact friction from $\mu_{a}=0.18$ to 0.36 , the slip-line field is expanded generally, but the extrusion zone CBD becomes narrower horizontally and deeper.

Fig. 10(a) and (b) show the variation of bearing capacity factor $N_{c}$ and that of $N_{q}$ with the pipeline foundation embedment $e_{0} / r$ for various values of the apparent interfacial friction angle (e.g., $\mu_{a}=0.18,0.36$ ), respectively. In this parametric study, the internal friction angle of the soil is chosen as $\phi=30^{\circ}$. As shown in Fig. 10 (a) and (b), both $N_{c}$ and $N_{q}$ increases monotonically with the increase of $e_{0} / r$ in the range of $0 \leq e_{0} / r \leq 1.0$. The curves are approximately parallel to each other for various values of the apparent interfacial friction angle. As aforementioned in Section 2.1, for the case of the pipeline embedment is larger than the pipeline's radius $\left(e_{0} / r>1.0\right)$, the pipeline embedment can be treated as $e_{0} / r=1$ with an equivalent uniform surcharge pressure $q=\gamma^{\prime}\left(e_{0}-r\right)$. That is, both of the bearing capacity factors are constant, whose values are dependent on the interfacial friction.

For better understanding bearing capacity of the rough pipeline foundation, the effects of pipe-soil interfacial friction on the dimensionless collapse load $P_{u} /$ cris further investigated. Under the conditions of weightless soil $\left(\gamma^{\prime}=0\right)$ and no surcharge $(q=0)$, the variations of $P_{u} / c r$ with $e_{0} / r$ for various values of $\mu_{a}$ are shown in Fig. 11. It is indicated that, there exists a notable positive
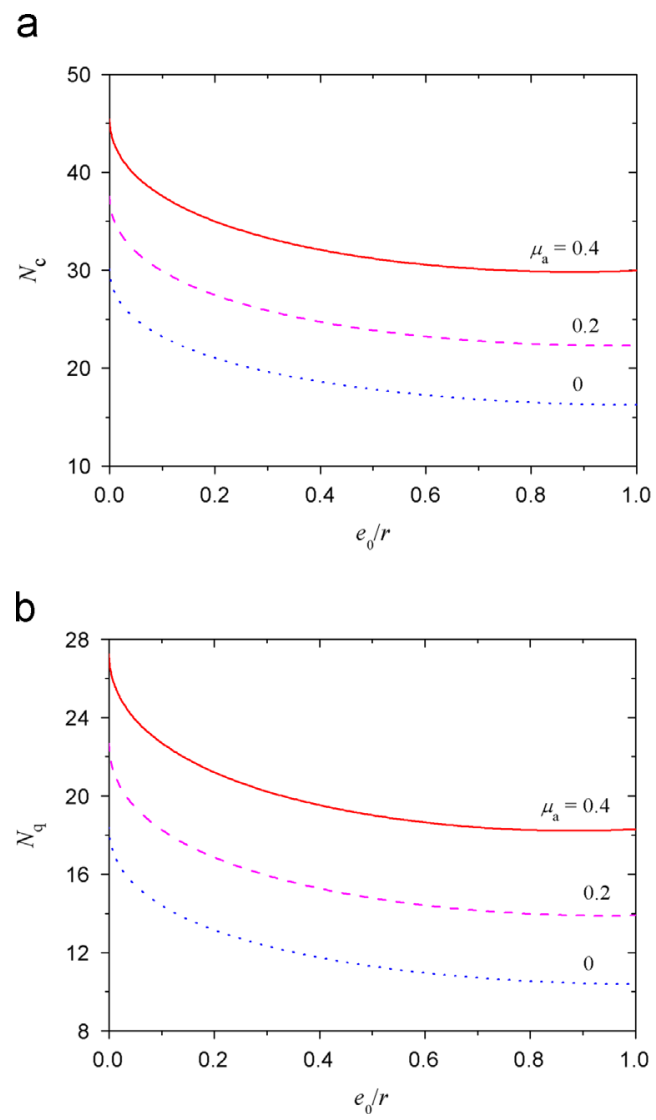

Fig. 10. Variation of bearing capacity factors with the pipeline foundation embedment for various values of the apparent interfacial friction angle: (a) $N_{c}-e_{0} / r$, (b) $N_{q}-e_{0} / r$. 


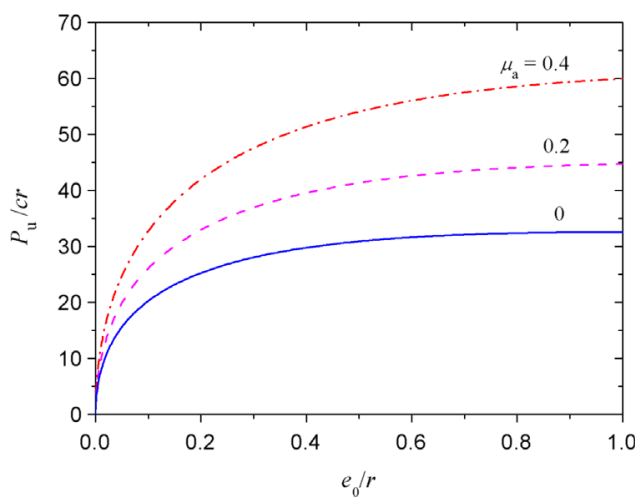

Fig. 11. Variations of $P_{u} / c r$ with $e_{0} / r$ for various values of $\mu_{a}$.

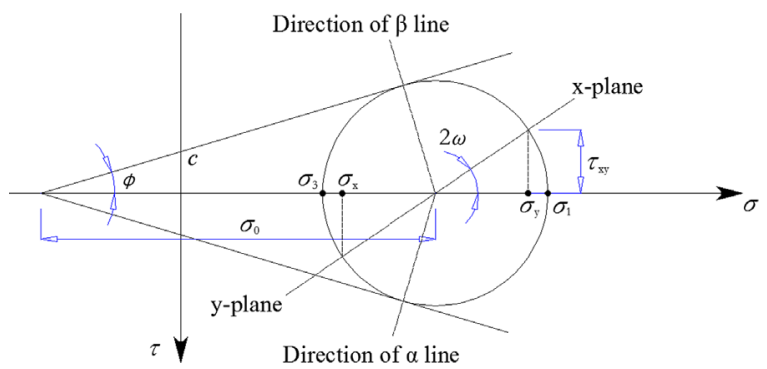

Fig. 12. Mohr circle for the point in the slip-line field obeying Mohr-Coulomb failure criterion.

correlation of the dimensionless collapse load with the pipeline embedment and the pipe-soil interfacial friction. Under the same pipe-soil interfacial friction condition, the collapse load $\left(P_{u} / \mathrm{cr}\right)$ increases gradually from zero to a relatively constant value with increasing the pipeline embedment from $e_{0} / r=0$ to 1.0. For a given value of the pipeline embedment $\left(e_{0} / r\right)$, the collapse load $\left(P_{u} / c r\right)$ increases greatly with increasing the pipe-soil interfacial friction $\left(\mu_{a}\right)$.

\section{Conclusion}

Unlike the previous analytical solutions for the bearing capacity of pipeline foundations, which mainly for the purely cohesive soils obeying Tresca criterion, a general slip-line field solution is derived for the ultimate bearing capacity of the pipeline on the drained soil obeying the Mohr-Coulomb criterion. Comparative study is then made for validation of the present slip-line field solution with numerical simulations. Moreover, based on the derived general slip-line field solution, parametric studies are made for better understanding the failure mechanism of the pipeline foundation. The conclusions are drawn as follows.

1. The derived slip-line field solution for the ultimate load of the pipeline foundation on Mohr-Coulomb soils matches well with the finite element simulations. The failure mechanism by the analytical solution is reasonably comparable to the incremental-displacement vector field simulated by utilizing finite element analysis.

2. As the internal friction angle of the soil approaches zero, the derived bearing capacity factors for the pipeline foundation obeying Mohr-Coulomb criterion can limit to those for a pipeline foundation obeying Tresca criterion previously derived by Gao et al. (2013).

3. Moreover, the derived bearing capacity factors for a fullysmooth pipeline limit to those for a conventional rectangular strip-footing while the pipeline embedment approaches zero. If the shallowly-embedded pipeline foundation is simply treated as a conventional strip-footing, the bearing capacity factors for the pipeline would be over evaluated.

4. The pipe-soil interfacial friction also has much effect on the bearing capacity of the pipeline foundation. The dimensionless collapse load increases with increasing the pipeline embedment and the pipe-soil interfacial friction.

\section{Acknowledgments}

This study was financially supported by the National Natural Science Foundation of China (Grant nos. 11372319, 11232012) and the Major State Basic Research Development Program of China (Grant no. 2014CB046204).

\section{Appendix A. Slip-line field theory: Mohr-Coulomb criterion}

The slip-line field theory is on the basis of equilibrium equations and the failure criterion of the material. In this study, the soil is assumed to obey Mohr-Coulomb failure criterion, which under the plane strain condition can be expressed as:

$$
\left(\frac{\sigma_{x}-\sigma_{y}}{2}\right)^{2}+\tau_{x y}^{2}=\left(\frac{\sigma_{x}+\sigma_{y}}{2}+c \cot \phi\right)^{2} \sin ^{2} \phi
$$

(A.1) can also be generally expressed with stress invariants (see Eq. (12)). The stress components in the soil obeying MohrCoulomb failure criterion (i.e. in the slip-line field) can be written as follows:

$$
\begin{aligned}
& \sigma_{x}=\sigma_{0}-c \cot \phi-\sigma_{0} \sin \phi \cos 2 \omega, \\
& \sigma_{y}=\sigma_{0}-c \cot \phi+\sigma_{0} \sin \phi \cos 2 \omega, \\
& \tau_{x y}=-\sigma_{0} \sin \phi \sin 2 \omega,
\end{aligned}
$$

where $x$ and $y$ are the coordinates for the point in the slip-line field; the positive directions of the stress components are shown in Fig. 9; $\omega$ is the angle from the $x$-axis to the first principal stress plane in the clockwise direction (see Fig. 12).

For a plane strain problem, the equilibrium equations are as follows:

$\frac{\partial \sigma_{x}}{\partial x}+\frac{\partial \tau_{x y}}{\partial y}=0$,

$\frac{\partial \tau_{x y}}{\partial x}+\frac{\partial \sigma_{y}}{\partial y}=0$

Submitting Eqs. (A.2a)-(A.2c) into Eqs. (A.3a) and (A.3b), the following equations can be obtained:

$$
\begin{aligned}
& (1-\sin \phi \cos 2 \omega) \frac{\partial \sigma_{0}}{\partial x}-\sin \phi \sin 2 \omega \frac{\partial \sigma_{0}}{\partial y} \\
& \quad+2 \sigma_{0} \sin \phi \sin 2 \omega \frac{\partial \omega}{\partial x}-2 \sigma_{0} \sin \phi \cos 2 \omega \frac{\partial \omega}{\partial y}=0
\end{aligned}
$$

$-\sin \phi \sin 2 \omega \frac{\partial \sigma_{0}}{\partial x}+(1+\sin \phi \cos 2 \omega) \frac{\partial \sigma_{0}}{\partial y}$

$-2 \sigma_{0} \sin \phi \cos 2 \omega \frac{\partial \omega}{\partial x}-2 \sigma_{0} \sin \phi \sin 2 \omega \frac{\partial \omega}{\partial y}=0$.

The characteristic equation for Eqs. (A.4a) and (A.4b) is

$\operatorname{det}\left(a_{\mathrm{ij}} d y-b_{\mathrm{ij}} d x\right)=0$, 
In which, the coefficient determinants $a_{\mathrm{ij}}$ and $b_{\mathrm{ij}}$ are

$a_{\mathrm{ij}}=\left(\begin{array}{cc}1-\sin \phi \cos 2 \omega & 2 \sigma_{0} \sin \phi \sin 2 \omega \\ -\sin \phi \sin 2 \omega & -2 \sigma_{0} \sin \phi \cos 2 \omega\end{array}\right)$,

$b_{\mathrm{ij}}=\left(\begin{array}{ccc}-\sin \phi \sin 2 \omega & -2 \sigma_{0} \sin \phi \cos 2 \omega \\ 1+\sin \phi \cos 2 \omega & -2 \sigma_{0} \sin \phi \sin 2 \omega\end{array}\right)$.

The characteristic Eq. (A.5) can be further expressed as:

$[d y / d x-\tan (\omega+\pi / 4+\phi / 2)] \cdot[d y / d x-\tan (\omega-\pi / 4-\phi / 2)]=0$,

where $d y / d x=\tan (\omega+\pi / 4+\phi / 2)$ and $d y / d x=\tan (\omega-\pi / 4-$ $\phi / 2$ ), are the characteristic functions for $\alpha$ line and for $\beta$ line, respectively. The geometrical characteristics of the slip-lines indicated by (A.6) can be figured with Figs. 9 and 12. The differential along $\alpha$ line and that along $\beta$ line are

$\frac{\partial}{\partial S_{\alpha}}=\cos (\omega+\pi / 4+\phi / 2) \frac{\partial}{\partial x}+\sin (\omega+\pi / 4+\phi / 2) \frac{\partial}{\partial y}$,

$\frac{\partial}{\partial S_{\beta}}=\cos (\omega-\pi / 4-\phi / 2) \frac{\partial}{\partial x}+\sin (\omega-\pi / 4-\phi / 2) \frac{\partial}{\partial y}$,

where $S_{\alpha}$ and $S_{\beta}$ represent the length of the arc along $\alpha$ and $\beta$ line, respectively. Set

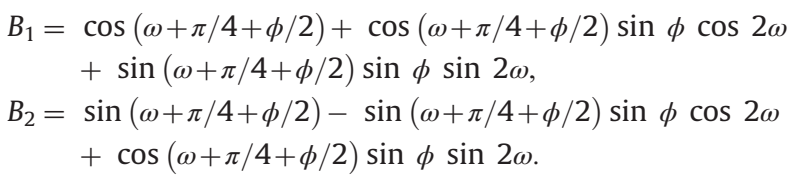

Then, Eq. (A.4a) multiplied by $B_{1}$ plus Eq. (A.4b) multiplied by $B_{2}$ makes

$$
\begin{aligned}
& \cos ^{2} \phi\left[\frac{\partial \sigma_{0}}{\partial x} \cos (\omega+\pi / 4+\phi / 2)+\frac{\partial \sigma_{0}}{\partial y} \sin (\omega+\pi / 4+\phi / 2)\right] \\
& +2 \sigma_{0} \frac{\partial \omega}{\partial x}[\sin \phi \sin 2 \omega \cos (\omega+\pi / 4+\phi / 2) \\
& \left.+\left(\sin ^{2} \phi-\sin \phi \cos 2 \omega\right) \sin (\omega+\pi / 4+\phi / 2)\right] \\
& -2 \sigma_{0} \frac{\partial \omega}{\partial y}\left[\left(\sin ^{2} \phi+\sin \phi \cos 2 \omega\right) \cos (\omega+\pi / 4+\phi / 2)\right. \\
& +\sin \phi \sin 2 \omega \sin (\omega+\pi / 4+\phi / 2)]=0 .
\end{aligned}
$$

Eq. (A.8) can be further simplified as:

$$
\begin{aligned}
& \cos ^{2} \phi\left[\frac{\partial \sigma_{0}}{\partial x} \cos (\omega+\pi / 4+\phi / 2)+\frac{\partial \sigma_{0}}{\partial y} \sin (\omega+\pi / 4+\phi / 2)\right] \\
& \quad-2 \sigma_{0} \sin \phi \cos \phi\left[\frac{\partial \omega}{\partial x} \cos (\omega+\pi / 4+\phi / 2)+\frac{\partial \omega}{\partial y} \sin (\omega+\pi / 4+\phi / 2)\right]=0 .
\end{aligned}
$$

Referring to Eq. (A.7a), then the following stress equation along the $\alpha$ line can be derived as:

$\frac{1}{\sigma_{0}} d \sigma_{0}=2 \tan \phi d \omega$.

Take the integral of Eq. (A.10) with respect of $\sigma_{0}$ and $\omega$ :

$\sigma_{0} e^{-2 \omega \tan \phi}=$ const $1 \quad$ (along $\alpha$ line).

Similarly, the following stress equation along $\beta$ line can be derived as:

$\sigma_{0} e^{2 \omega \tan \phi}=$ const2 (along $\beta$ line).

\section{References}

Chen, W.F., Liu, X.L., 1990. Limit Analysis in Soil Mechanics. Elsevier Scientific Publishing Co., New York.

Det Norske Veritas, 2010. On-Bottom Stability Design of Submarine Pipelines. Recommended Practice, DNV-RP-F109.

Gao, F.P., Wang, N., Zhao, B., 2013. Ultimate bearing capacity of a pipeline on clayey soils: slip-line field solution and FEM simulation. Ocean Eng. 73, 159-167.

Gao, F.P., Zhao, B., 2012. Slip-line field solution for ultimate bearing capacity of a pipeline on clayey soils. Theor. Appl. Mech. Lett. 2, 051004.

Gourvenec, S., Randolph, M., 2003. Effect of strength non-homogeneity on the shape of failure envelopes for combined loading of strip and circular foundations on clay. Géotechnique 53 (6), 575-586.

Knappett, J.A., Craig, R.R.F., 2012. Craig's Soil Mechanics. Taylor \& Francis, London. Hansen, J.B., 1970. A Revised and Extended Formula for Bearing Capacity. Danish Geotechnical Institute, Copenhagen, pp. 5-11 (Bulletin No. 28).

Hibbitt, KarlssonandSorensen, 2006. ABAQUS Theory Manual, Version 6.5-1.

Hodder, M.S., Cassidy, M.J., 2010. A plasticity model for predicting the vertical and lateral behaviour of pipes in clay soils. Géotechnique 60 (4), 247-263.

Li, J., Tian, Y., Cassidy, M.J., 2015. Failure mechanism and bearing capacity of footings buried at various depths in spatially random soil. J. Geotech. Geoenviron. Eng. 141 (2), 04014099.

Potts, D.M., Zdravković, L., 1999. Finite Element Analysis in Geotechnical Engineering: Theory. Thomas Telford Ltd., London.

Potts, D.M., Zdravković, L., 2001. Finite Element Analysis in Geotechnical Engineering: Application. Thomas Telford Ltd., London.

McMahon, B.T., Haigh, S.K., Bolton, M.D., 2014. Bearing capacity and settlement of circular shallow foundations using a nonlinear constitutive relationship. Can. Geotech. J. 51, 995-1003.

Murff, J.D., Wagner, D.A., Randolph, M.F., 1989. Pipe penetration in cohesive soil. Géotechnique 39 (2), 213-229.

Small, S.W., Tambuvello, R.D., Piaseckyj, P.J., 1971. Submarine pipeline support by marine sediment. In: Proceedings of the Annual Offshore Technology Conference, Houston TX, Paper OTC-1357, pp. 309-318.

Tian, Y., Cassidy, M.J., 2008. Modelling of pipe-soil interaction and its application in numerical simulation. Int. J. Geomech. 8 (4), 213-229.

Tian, Y., Cassidy, M.J., Gaudin, C., 2010. Advancing pipe-soil interaction models through geotechnical centrifuge testing in calcareous sands. Appl. Ocean Res. 32 (3), 284-297.

Zhang, J., Stewart, D.P., Randolph, M.F., 2002. Modelling of shallowly embedded offshore pipelines in calcareous sand. J. Geotech. Geoenviron. Eng. 128 (5), 363-371. 